\title{
Metabolite Ratio AUC Last Nonzero Concentration
}

National Cancer Institute

\section{Source}

National Cancer Institute. Metabolite Ratio AUC Last Nonzero Concentration. NCI

Thesaurus. Code C156567.

The ratio of AUC to last nonzero concentration for the metabolite to the AUC to last nonzero concentration for the parent analyte. 